\title{
Microalbuminuria as a Marker of Preclinical Diastolic Dysfunction in Newly Diagnosed and Never-Treated Essential Hypertension
}

\section{Gajurel R M , Sayami A}

Department of Cardiology, Tribhuvan University, Man Mohan CardioThoracic Vascular and Transplant Centre, Maharajgunj, Kathmandu, Nepal

Correspondence address: Dr Ratna Mani Gajurel, Department of Cardiology, MCVTC, Maharajgunj.

Email: ratnamanigajurel@gmail.com

\begin{abstract}
Introduction: Hypertension (HTN) is a global public health problem with one fourth adults worldwide estimated to have high blood pressure (BP $)^{1}$ The incidence of hypertension continues to increase in all developed and developing societies as the population grows older and more obese. ${ }^{2}$ The Framingham Study and other epidemiological surveys have clearly defined HTN as an important cause of morbidity and mortality. ${ }^{2-4}$ The aim of this study was to determine the spot urine Microalbuminuria as a marker of preclinical cardiac structural and functional changes in the form of left ventricular diastolic dysfunction in newly diagnosed and never treated essential hypertensive subjects.

Methods: A cross sectional study was used for those patients who were attended outpatient clinic of MCVTC with diagnosis of newly diagnosed and never treated hypertension over a period of October 2011 to November 2012.

Results: Atotal of 130 essentialhypertensive patients underwent for spoturine formicroalbuminuria estimation and Echocardiography for evaluation of left ventricular diastolic function. Among 56 (43.1\%) urine samples showed negative test [(Microalbuminuria -); ( UACR $30 \mathrm{mg} / \mathrm{Gm}$ )] and those $74(56.9 \%)$ samples revealed positive test [(Microalbuminuria+); (UACR 30 to $300 \mathrm{mg} / \mathrm{Gm})$ ]. Patients with microalbuminuria positive was found to have more left ventricular diastolic dysfunction than those who were negative for Microalbuminuria.

Conclusion: Microalbuminuria was found to have early preclinical marker of myocardial dysfunction in the form of left ventricular diastolic dysfunction in new and never treated essential hypertension.
\end{abstract}

Key words: Hypertension, Microalbuminuria, Left Ventricular diastolic dysfunction

\section{Introduction}

The first scientific HTN survey in Nepal was done in $1981 .^{5}$ The prevalence of HTN according to old World Health Organization (WHO) criteria $(160 / 95 \mathrm{mmHg})$ in the various parts of the country was $5.3 \%$ in Jumla to $10 \%$ in urban Kathmandu. ${ }^{6}$ A study from Eastern Nepal in 2005 found a prevalence of almost $23 \%$ according to the Jet Navigation Chart (JNC) VII guidelines. ${ }^{7}$ In a Teaching Hospital based observation in Nepal, $3.9 \%$ of the admitted patients were suffering from HTN. ${ }^{8}$ In a descriptive hospital based study conducted in 3 central hospitals of Nepal, complications of hypertension were ranges from $16.0 \%$ to $36.0 \%$. To prevent various complications, hypertension demands proper diagnosed and adequate treatment. ${ }^{9}$ According to World Health Report (2003), cardiovascular disease was responsible for 16.7 millions of the total global deaths and 3.9 millions only from hypertension. Around $80 \%$ of cardiovascular deaths took place in low and middle-income countries. ${ }^{10}$ According to Nepal Health research council (NHRC), a hospital based study done in 2010 showed among Cardiovascular diseases, prevalence of hypertension 
was $47 \%$ followed by Cerebrovascular accident, Congestive cardiac failure, Ischemic heart disease, Rheumatic heart disease and Myocardial Infarction in Nepalese population which is similar as that of WHO report in South East Asia Region. It reflects that Nepal is also facing the surging burden of hypertension similar to other developing nations of South East Asia and trend towards global pandemic of Hypertension. ${ }^{11}$

Many international studies showed the heavy burden of hypertension and its complications are in low and middle income countries. Till now no organized studies on hypertension and its complications have been conducted in Nepal. So the clinical implications of microalbuminuria as a marker of preclinical left ventricular diastolic dysfunction in Nepalese hypertensive community are largely unknown.

The present study has attempted to answer above question. This study is designed to know the preclinical complications of hypertension in tertiary care hospital of Nepal. The aim of this study is to determine the spot urine Microalbuminuria as a marker of preclinical cardiac structural and functional changes in the form of left ventricular diastolic dysfunction in newly diagnosed and never treated essential hypertensive subjects. The entire study subject had undergone baseline investigations including spot urine for microalbumin estimation and echocardiography to assess left ventricular diastolic function in outpatient basis. This study was limited only to outpatient, newly diagnosed and untreated hypertension to minimize the study bias by excluding chronic hypertension and its overt complications.

\section{Methods}

This is a cross sectional study and ethical clearance was obtained from the Institutional Review Board of the Research Department of Institute of Medicine. Newly diagnosed and never treated hypertension cases attended in the outpatient clinics of MCVTC were included in the study. Data were taken by the investigator by preformed questionnaire over a period of October 2011 to November 2012. The data were entered in SPSS statistical analysis tool and were descriptively analyzed. Patients who already had history of hypertension more than two years, with complications, other comorbidities and extreme of ages were not included in the study. The eligible patients enrolled for the study was otherwise healthy and aged $30-55$ years.

\section{Results}

A total of 130 patients enrolled in the study, predominantly $74(56.9 \%)$ were male and $56(43.1 \%)$ were female and the maximum numbers of patients were from between age group 35 to 50 years. Most of these patients were from Kathmandu Valley and majorities were literate to well educated

Almost all the patient were asymptomatic and new or never treated hypertension [(Mean SBP $155.1846 \mathrm{mmHg}$ $\pm 10.998 \mathrm{mmHg}) ;($ DBP $95.2 \mathrm{mmHg} \pm 5.543 \mathrm{mmHg})]$ and presented to doctors because either they have significant family history $[46.2 \%(60)]$ or found to have hypertension on regular check up. All the patients were clinically normal during examination shown in table 1.

Table1: Level of blood pressure

\begin{tabular}{llllllll} 
Blood Pressure & Minimum & Maximum & Range & Mean & SE Mean & \multicolumn{2}{c}{ Median SD } \\
SBP Right Arm & 140 & 200 & 60 & 155.18 & .96460 & 155.00 & 10.998 \\
SBP Left Arm & 140 & 200 & 60 & 152.53 & .99529 & 150.65 & 11.348 \\
DBP Right Arm & 90 & 110 & 20 & 95.20 & .48623 & 95.00 & 5.543 \\
DBP Left Arm & 90 & 110 & 20 & 93.61 & .44700 & 92.70 & 5.096
\end{tabular}

The selected 130 patients' microalbuminuria was estimated from urinary albumin creatinine ratio from the early morning random urine samples without any preservatives by immunoturbidimetric method. Among them 56 (43.1\%) urine samples showed negative test for microalbumin [(Microalbuminuria -);( UACR $30 \mathrm{mg} / \mathrm{Gm})$ ] and remaining 74 (56.9\%) samples revealed positive test for microalbumin [(Microalbuminuria+);(UACR 30 to $300 \mathrm{mg} / \mathrm{Gm})$ ] as dipicted in figure 1 . 


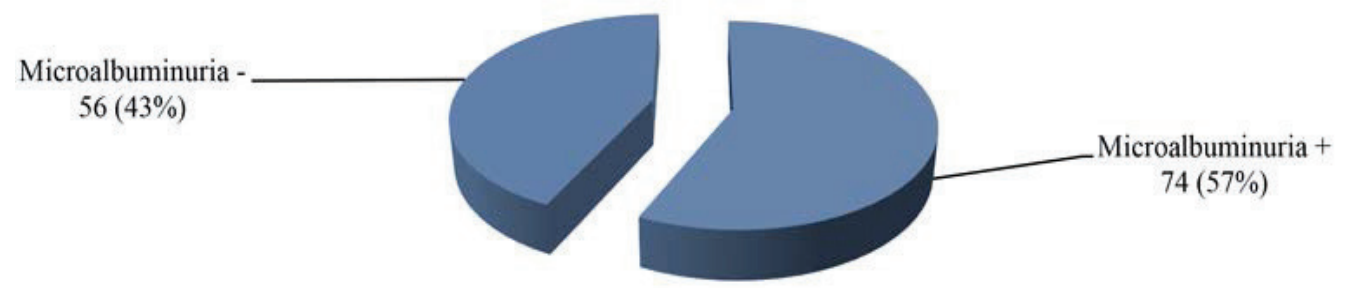

Figure 1 Microalbuminuria among hypertension patients

Among 130 hypertension cases 56 (43.1\%) had echocardiography findings of Normal LV Diastolic function, 22 (16.9\%) patients had Grade I and 50 (38.5\%) patients had Grade II LV Diastolic Dysfunction and 2 (1.5\%) patient had Grade IIIa LV Dysfunction. $(\mathrm{P}<0.01)$ Figure:2 and individual indices of left ventricular diastolic function are shown in table 2.

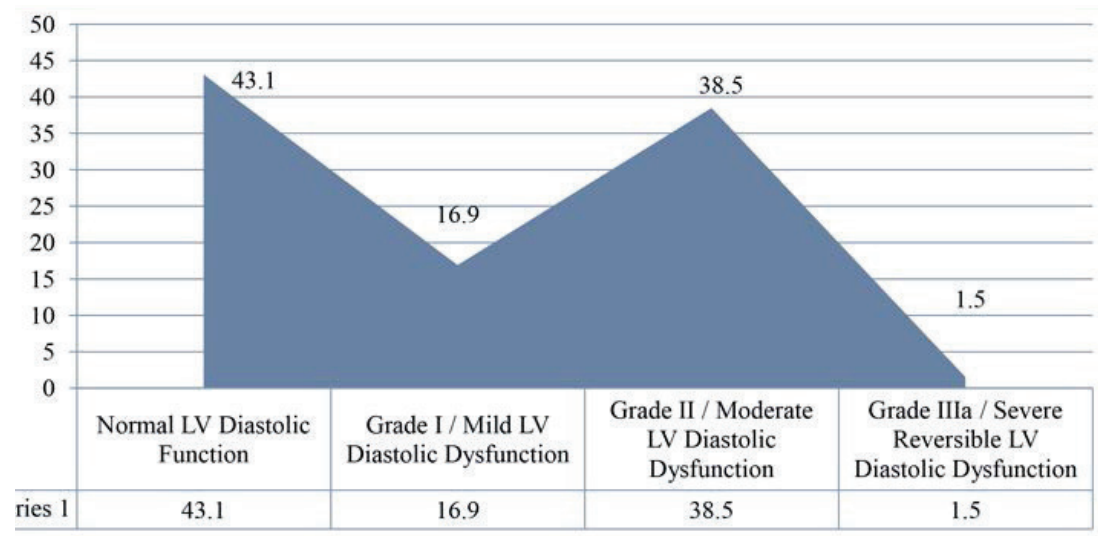

Figure 2 Final Echocardigraphic Impression On Left Ventricular Diastolic Function

Table 2: Relation of Different LV diastolic indices to LV Diastolic Function

\begin{tabular}{lllllll} 
LV Diastolic Function & E/A Ratio & Annular & IVRT & DT & E/E' & Vp \\
N=130 & & E Velocity & & & & \\
\hline Normal LV Diastole & 54 & 46 & 38 & 42 & 40 & 64 \\
Grade I LVDD & 28 & 34 & 24 & 36 & 28 & 22 \\
Grade II LVDD & 46 & 42 & 54 & 40 & 56 & 42 \\
Grade IIIa LVDD & 2 & 8 & 14 & 12 & 6 & 2
\end{tabular}

In echocardiography study of left ventricular diastolic function, among Microalbuminuria negative hypertensive cases [(N=56/130) 43.1\%] we found 52(40\%) had Normal LV Diastolic function, [N=2/130 (1.5\%)] each (total 3\%) had Grade I and II LV Diastolic Dysfunction and no patient was shown to have Grade III LV Dysfunction which is statistically significant. $(\mathrm{P}<0.01)$

Out of 130 patients, $[\mathrm{N}=74 / 130(56.9 \%)]$ had Microalbuminuria positive. Among them only [N=4/130 (3\%)] patients were found to have Normal LV Diastolic function and rest $[\mathrm{N}=70 / 130]$ had different types of left ventricular diastolic Dysfunction (LVDD). Among LVDD [N=20/130 (15.38\%)] patients had found to have Grade I LVDD, [N=48/130 $(36.92 \%)$ ] patients had Grade II LVDD and [N=2/130 (1.5\%)] patients were found to have Grade IIIa LVDD which is statistically significant. $(\mathrm{P}<0.05)$ Graph:1, Flow Chart:1 
Flow chart 1 Result of Urine for Microalbumin with LV Diastolic dysfunction in Hypertension.

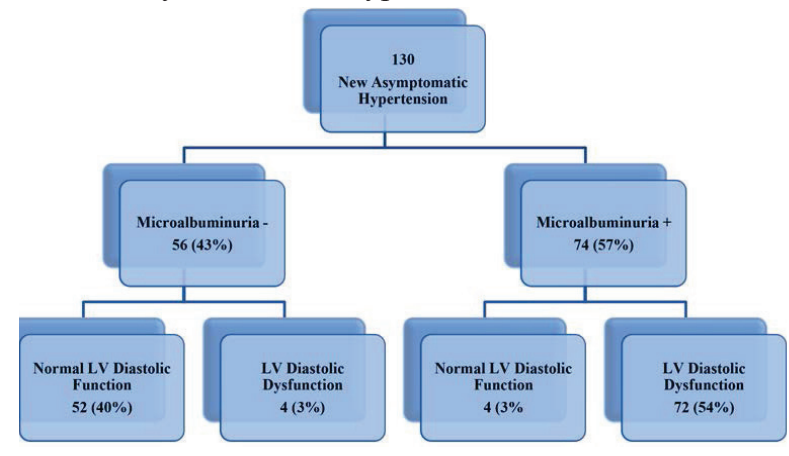

On subgroup analysis among Microalbuminuria Negative Group out of $56[\{\mathrm{~N}=52 / 56\}(92.85 \%)]$ patients were found to have normal diastolic function; $[\{\mathrm{N}=2 / 56\}$ $(3.57 \%)]$ patients had Grade I LVDD and $[\{\mathrm{N}=2 / 56\}$ $(3.57 \%)$ ] patients had Grade II LVDD. No patient was found to have Grade III LVDD. Similarly subgroup analysis among the Microalbuminuria Positive Group out of 74 patients, only $\quad[\{\mathrm{N}=4 / 74\}(5.4 \%)]$ had normal LV Diastolic Function; $[\{\mathrm{N}=20 / 74\}(27 \%)]$ were found to have Grade I LVDD; [ $\{\mathrm{N}=48 / 74\}(64.86 \%)]$ had Grade II LVDD and $[\{\mathrm{N}=2 / 74\}(2.7 \%)]$ had Grade IIIa LVDD. (P $<0.05)$ as depicted in flow chart 2
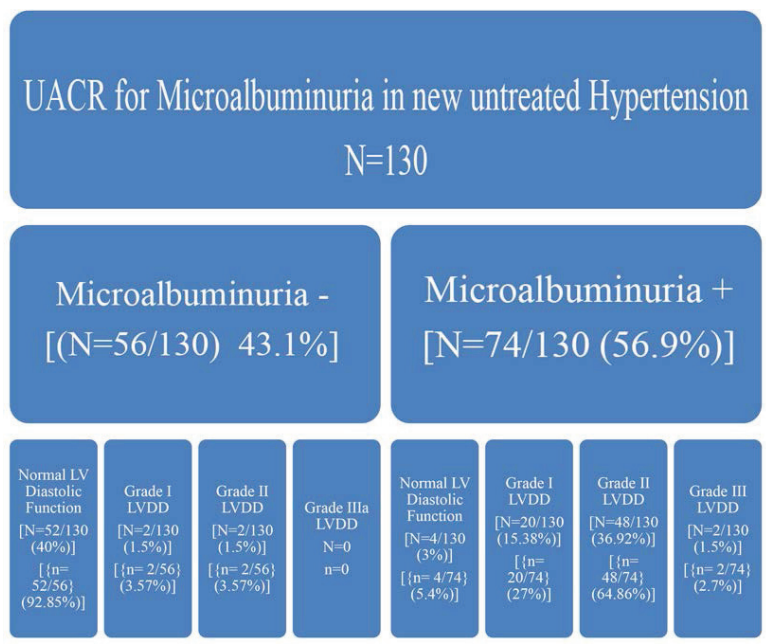

\section{Flow Chart 2}

In our study cardiac mass was interpreted by comparing cardiomegaly in CXR [N=18/130, (13.8\%)], LVH in 12 lead ECG $[\mathrm{N}=26 / 130,(20 \%)]$ and Echocardiographic increase in LV mass in $[n=49 / 130,(37.7 \%)]$ and Concentric LVH $[\mathrm{N}=53 / 130 ;(40.8 \%)]$ respectively. The LV mass index was greater in those with microalbuminuria positive cases; as was the prevalence of LV hypertrophy. UACR was directly correlated with both echocardiographic indices of LV diastolic function and changes in LV geometry. $(\mathrm{P}<0.01)$ Figure: 4

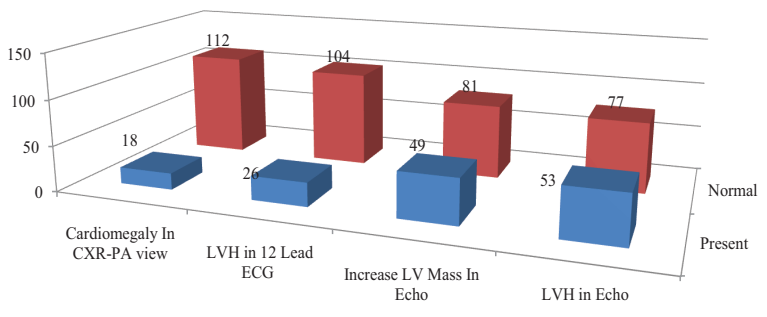

Figure 4 Cardiac Mass Shown by Different Indices

\section{Discussion}

Systemic hypertension and its complications are responsible for a large portion of the hospital admissions worldwide, representing a very high socioeconomic cost annually. Cardiovascular diseases are responsible for the major death as well, with a direct relation between cardiovascular mortality and blood pressure levels. Hypertension is considered the most common and the importance cardiovascular morbidity in general adult population. ${ }^{12}$

Many recent studies demonstrated that microalbuminuria is also associated with worse cardiovascular outcomes in hypertension and, even in the general population. Microalbuminuria is the first clinical sign of involvement of kidney. Parving et al, in 1974 was the first to report microalbuminuria in hypertensive patients without diabetes. Since then, several studies have shown the true prevalence of microalbuminuria in essential hypertension varies in the range $20-40 \%$, according to the selection criteria used. ${ }^{2,3}$ The subanalysis of the Swiss and German centers of the global i-SEARCH survey generated the prevalence of MAU in clinical cardiology (53.1\%) in comparison to most of the previous studies in similar populations. Another population based study had evidence of MAU (60.2\%) with prevalence rates higher in men $(57.0 \%)$ than women (43.0\%).

As the MAPS (Microalbuminuria Prevalence Study in hypertensive patients) is the first large multicentre epidemiological study conducted in Asia, this sub analysis of data from Pakistan and Thailand indicates that 24.2\% of the analyzed patients had microalbuminuria. The prevalence of microalbuminuria was slightly higher than the rates of $17 \%$ to $21 \%$ reported from western populationbased studies in patients with diabetes. Overall in this study of ten Asian countries, the prevalence of microalbuminuria was $39.8 \%$ and the prevalence of macroalbuminuria was 
$18.8 \%$. The highest prevalence of microalbuminuria was observed in Korea (56.5\%) and the lowest in Pakistan $(24.2 \%)$. In the MAPS study subanalysis of overall ten Asian countries, the prevalence of microalbuminuria was $39.8 \%$ and the prevalence of macroalbuminuria was $18.8 \%$. The highest prevalence of microalbuminuria was observed in Korea $(56.5 \%)$ and the lowest in Pakistan $(24.2 \%) .{ }^{13}$

In our study in comparison to other studies microalbuminuria was more frequent in younger age group ( $<50$ years). This may be because our eligible patients for study are relatively young and majority fall between age 35 to 50 years and prevalence of Microalbuminuria is $56.9 \% \quad(\mathrm{p}<0.01)$ correlated with the other international studies.

Diastolic dysfunction is a risk factor for the development of cardiovascular morbidity, and has prognostic value in population settings. Diastolic dysfunction is very frequent and is actually sign of manifest heart weakness. Many of patients with heart weakness have isolated left ventricular diastolic dysfunction (LVDD). New diagnostics methods as Doppler Echo with close monitoring enables precise and early LVDD diagnose so we tried to utilize the role of echocardiography in our study.

In our study, we demonstrated that in a hospital based hypertensive patients without overt cardiac disease, diabetes, and other comorbidities which have an independent negative impact on LV diastolic function were excluded from the study. We showed that hypertension were independently associated with a higher $\mathrm{E} / \mathrm{E}^{\prime}$ ratio, an index of LV end diastolic pressure, even after adjustment for covariates that significantly affect diastolic function such as age, LVM, geometry, heart rate, and LVEF. The finding of a higher LV end-diastolic pressure in hypertension, could explain in part the additional risk of developing LV Diastolic Dysfunction and diastolic heart failure in patients.

In this respect, the exclusion, from our study, of subjects with evidence of coronary artery disease or with abnormal LV systolic function allowed us to derive important information on the effect of the most prevalent cardiovascular risk factors on diastolic function at early stages of disease. The prevalence of diastolic dysfunction was higher in Hypertension with microalbuminuria than in hypertension without microalbuminuria. (56.9\% Vs $43.1 \%$ ). When we considered the proportion of subjects with elevated LV end-diastolic pressure (identified by an $E / E^{\prime}$ ratio), we found it to be significantly greater in the hypertension with microalbuminuria group (56.9\%) than in Hypertension without microalbuminuria $(43.1 \%)$. We found that the group with hypertension and microalbuminuria had significantly more diastolic function than the microalbuminuria negative group. E' velocity was lower in microalbuminuria negative group whereas the $\mathrm{E} / \mathrm{E}^{\prime}$ ratio was significantly higher in microalbuminuria positive group suggesting an effect of microalbuminuria in hypertension and on the LV end-diastolic pressure as an independent variable to predict the result. However, although the E/A ratio showed a trend toward lower values in patients with hypertension and pseudo normalization pattern and which is adjusted with other parameter of diastolic indices. This observation may appear surprising, but it is consistent with the pathophysiology of the LV diastolic dynamics. The E/A ratio is strongly dependent on cardiac load, and follows a U-shaped curve in the natural history of LV diastolic dysfunction, with a reduction in the earlier stages of dysfunction. In this stage, the relaxation of the LV is delayed and the early diastolic flow (E-wave) becomes slower; therefore, as a compensatory mechanism, the atrial contribution to the LV filling increases, and the E/A ratio decreases (diastolic dysfunction, Grade I).With the progression of diastolic dysfunction, the increasing pressure gradient between the left atrium and the LV acts as a propelling force, causing an increase in the E-wave velocity. As a consequence, the E/A ratio increases as well, becoming indistinguishable from a normal flow pattern. In this regard, the analysis of other Doppler flowderived parameters (mitral isovolumetric relaxation time and E-wave deceleration time) that are load dependent is not useful for identifying the apparent reversal of the mitral inflow to a normal pattern (also known as 'pseudo normalization' of the mitral inflow) as actual progression of the disease (diastolic dysfunction, Grade II). The mitral annulus early diastolic velocity assessed by pulsed TDI (E') has been shown to be less pre-load dependent, and its decrease with the progressive impairment of the LV diastolic mechanics allows the identification of LV abnormal relaxation and reduced LV compliance, and can identify diastolic dysfunction even in the presence of a pseudo normal mitral flow.

The overall prevalence of diastolic dysfunction in the hypertension according to study based on adult hypertensive nigerians published in ghana medical journal was $82.86 \%$. Prevalance of LV Diastolic Dysfunction in Hypertensives in Europe had $46 \%-48 \%$. The works of Kingue et al in Cameroon, and Balogun and Co-workers in Nigeria earlier referred to, documented $67.4 \%$ and $58 \%$ prevalence, respectively. Prevalence of LVDD in study based on London by Mayet et al was found to have $68 \%$. Work of Philips et al rather discovered abnormal left ventricular filling rates only in patients with systolic blood pressure (SBP) higher than $130 \mathrm{mmHg}$, but not in those patients with SBP lower than $130 \mathrm{mmHg}$. 
It would be noted that in our study the prevalence rate of LVDD for the patients with new untreated HTN (56.9\%) approximated and well correlated with above studies. On analysis of 130 subjects our study with untreated and newly diagnosed Essential Hypertension who underwent Urine for Microalbumin estimation and Echocardiographic LV Diastolic evaluation, it revealed final impression of Microalbuminuria as a Preclinical or Early predictor of myocardial dysfunction that can be quantify by Echocardiography which is comparable with many of the studies done in different part of the world including US, Europe and Asian countries. In this study out of 130 patients $56(43.1 \%)$ patients had Microalbuminuria - and $74(56.9 \%)$ patients had Microalbuminuria +. Findings of our study is comparable with MAPS and i-SEARCH studies which were done in Asian and European population respectively ${ }^{13,14,15}(\mathrm{P}<0.05)$.

\section{Conclusion}

In newly diagnosed and never-treated hypertensive patients, microalbuminuria is not only associated with greater myocardial mass, but is also related with preclinical impairment of LV diastolic function. This relationship of increased BP, increased LV mass and change in LV geometry and increase LV Diastolic Dysfunction as shown in our study, strengthens the role of microalbuminuria as an early and reliable marker of preclinical cardiac structural and functional dysfunction. This study also further confirmed that microalbuminuria is a powerful predictor of high cardiovascular risk in preclinical stage who is otherwise healthy newly diagnosed as well as never treated essential hypertensive patients. It is strongly recommended that all the patients with hypertension once the diagnosis is made, should undergo these two noninvasive tests as screening tests for early detection of anticipated complications.

Conflict of interest: The authors declare that they have no conflict of interests.

\section{References}

1. Kearney PM, Whelton M, Reynolds K, et al. Global burden of hypertension: analysis of the worldwide data. Lancet 2005; 365:217-23.

2. Domanski M, Mitchell G, Pfeffer M et al. Pulse pressure and cardiovascular disease-related mortality: Followup study of the Multiple Risk Factor Intervention Trial (MRFIT). J Amer Med Assoc 2002; 287: 2677-83.

3. Lewinston S, Clarke R, Oizilbash $\mathrm{N}$ et al. Age-specific relevance of usual blood pressure to vascular mortality: A meta-analysis of individual data for one million adults in 61 prospective studies. Lancet 2002; 360: 1903-13.
4. World Health report. Geneva, WHO,1997.

5. Vaidya A, Pathak R P, Pandey M R. Prevalence of hypertension in Nepalese community triples in 25 years:a repeat cross-sectional study in rural Kathmandu, Indian Heart Journal 2012; 64:128-131

6. Pandey MR, Dhungel S. Prevalence of hypertension in an urban community of Nepal. JNMA 1983; 21:1-5.

7. Vaidya A, Pokharel PK, Nagesh S, et al. War veterans of Nepal and their blood pressure status: a populationbased comparative study. J Hum Hypertension 2007; 21:900-3.

8. Sayami A, Shrestha B. Critical Care: Manual of ICU and CCU TU Teaching Hospital 1st ed. Kathmandu: JICA Med Edu Project 1995; 1-11.

9. Bista B. Factors associated with the hypertensive complications among the compliance to treatment regimen. (abs). Nepal health research council/ World Health Organization. Kathmandu. Bibliogrphic Database Health Res Reports in Kathmandu Valley 2004; Vol 3: MFN 0438.

10. WHO. Non Communicable Diseases. [Cited 201011 August]; Available from: http://www.searo.who.int/en/ Section1174/Section1459_7409.htm.

11. Nepal health research council, Prevalence of noncommunicable disease in Nepal ,A hospital based study, 2010

12. Stamm et al Systemic hypertension and roalbuminuria, Arq Bras Cardiol 2007; 89(6): 376-381

13. Peera Buranakitjaroen, Chaicharn Deerochanawong, Pongamorn Bunnag Microalbuminuria Prevalence Study (MAPS) in hypertensive Patients with Type 2 Diabetes in Thailand, J Med Assoc Thai 2005; 88: 11.

14. Muhammad Yakoob Ahmadani, Asher Fawwad, Abdul Basit, Zafar Iqbal Hydrie microalbuminuria prevalence study in hypertensive patients with type 2 diabetes in Pakistan, J Ayub Med Coll Abbottabad 2008; 20(3)

15. Ulrich Tebbea, Peter Bramlageb, Martin Thoenesc,d, W. Dieter Paard, Nicolas Danchine, Massimo Volpef, Jochen Schraderg, Georg Nollh, Michael Burnieri, Michael Böhm , Prevalence of microalbuminuria and its associated cardiovascular risk: german and swiss results of the recent global i-search survey, Swiss med wkly 2009; 139: 473 - 480 . 